\title{
Spontaneous rupture of an unscarred uterus with an intact amniotic sac extrusion and fetal leg entrapment at 28 gestational weeks: a case report
}

\author{
Wen-Xi Tan ${ }^{1}$, Xue-Jiao Lv $^{2}$, Rui-Qi Yang ${ }^{1}$, Ben-Zheng Zhao ${ }^{1}$, Jun-Yu Chen ${ }^{1}$, Shan Wu ${ }^{1}$, Yan-Hui Zhao ${ }^{1}$, \\ Man-Hua Cui ${ }^{1}$ \\ ${ }^{1}$ Department of Obstetrics and Gynecology, The Second Hospital of Jilin University, Changchun 130041, China; ${ }^{2}$ Department of Respiratory, The \\ Second Hospital of Jilin University, Changchun 130041, China \\ Correspondence to: Man-Hua Cui; Yan-Hui Zhao. Department of Obstetrics and Gynecology, The Second Hospital of Jilin University, Ziqiang Road \\ 218st, Changchun 130041, China. Email: cuimanhua@126.com; 9177312@qq.com.
}

\begin{abstract}
The spontaneous rupture of an unscarred uterus at 28 gestational weeks is an extremely rare event, particularly when associated with an intact amniotic sac extrusion and fetal leg entrapment, which has not been previously reported. A 27-year-old primigravid woman was referred to our department, due to perpetual abdominal pain, at 28 weeks and 5 days of gestation. The patient, G3p0, had previously undergone two induced abortions. At the time of admission, abdominal ultrasonography suggested a defect in the left uterine horn. An emergency laparotomy was subsequently performed and revealed an intact amniotic sac extrusion and fetal leg entrapment. Considering the risk of placental abruption, and the possibility of a secondary rupture if the gestation was not terminated, an emergency Cesarean section was recommended. Uterine rupture may be suspected whenever a patient complains of durative abdominal pain at 28 weeks and 5 days of gestation, even in the absence of an intra-abdominal hemorrhage or vaginal bleeding.
\end{abstract}

Keywords: Case report; pregnancy; uterine rupture; misdiagnosis; digestive tract diseases

Submitted Nov 05, 2019. Accepted for publication Feb 12, 2020.

doi: $10.21037 /$ gs.2020.02.16

View this article at: http://dx.doi.org/10.21037/gs.2020.02.16

\section{Introduction}

Uterine rupture is defined as the full-thickness separation of the uterine wall and the overlying serosa (1). This condition represents a major obstetric complication that is associated with high rates of maternal morbidity and mortality (2). A multitude of factors increase the risk of unscarred uterine rupture, including grand multiparity, undiagnosed fetopelvic disproportion, malpresentation, macrosomia, oxytocin stimulation, macrosomic-hydrocephalic fetus, placenta previa-percreta, previous invasive mole and previous instrumental abortion (3). Neonatal and maternal survival following uterine rupture depends on a swift diagnosis and rapid intervention (4). A deceleration of the fetal heart rate (FHR) may represent the earliest and most sensitive indicator of uterine rupture. In cases of uterine rupture, prompt surgical intervention is critical in optimizing the maternal and fetal outcome (5). The most common presenting symptom of spontaneous rupture is sudden and severe abdominal pain, which may be associated with vaginal bleeding, shock and fever (6).

In developed countries, the incidence of uterine rupture is less than one in 1,000 deliveries, and in $92 \%$ of cases, uterine rupture is associated with a previous Cesarean delivery (7). The risk factors of spontaneous rupture of an unscarred uterus have been reported previously $(3,8)$. However, the antepartum rupture of an unscarred uterus with intact sac extrusion and fetal leg entrapment, occurring early in gestation, has not previously been reported.

Here, we present the case of a patient that involves the antepartum rupture of an unscarred uterus with intact sac extrusion and fetal leg entrapment of primiparity at 28 weeks and 2 days of gestation. 


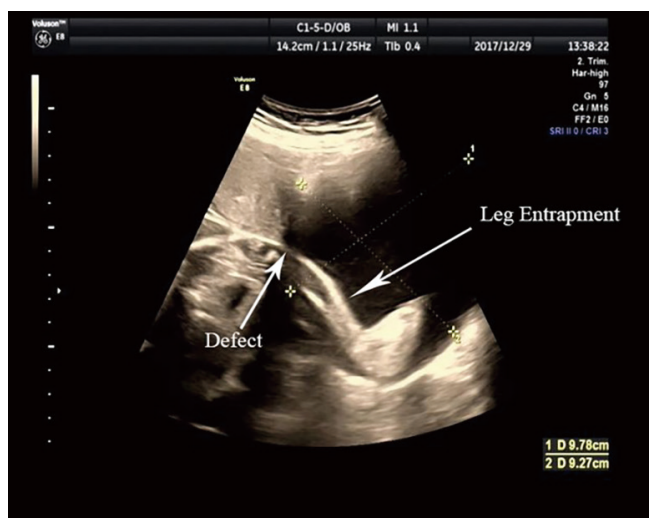

Figure 1 Ultrasound image showing the gestational sac protruding from the left uterine horn of the uterus (defect indicated by arrow); the contents of the protruding sac include the lower limbs of the fetus (leg entrapment indicated by arrow) and the amniotic fluid.



Figure 2 Photograph of the complete rupture of the left uterine uterus wall (approximately $10 \mathrm{~cm}$ in size) and the amniotic sac protruding from the rupture.

\section{Case presentation}

A 27-year-old primigravid woman, G3p0, at a gestational age of 28 weeks and 5 days, as determined by the last menstrual period, was referred to the Obstetrics and Gynecology Department of our hospital due to three days of sustained abdominal pain. According to ultrasonography, the local maternal and child health-care center considered the symptoms to be due to enterocinesia. Previously, the prenatal course of the patient had been uneventful and devoid of complications. She had been monitored with serial ultrasound scans by the medical department throughout the pregnancy and no fetal anomalies had been identified. There were no obvious maternal abnormalities during the pregnancy, such as abdominal pain, vaginal bleeding or fluid release.

The medical history of the patient was remarkable. She had experienced two previous abortion surgeries. It is commonly accepted that the surgical history of the patient is strongly associated with scar tenderness and that a history of uterine surgery is associated with a high risk of suffering from uterine rupture. In the most recent literature review of cases involving uterine rupture, the majority of uterine ruptures were found to occur in women who had a history of previous uterine surgeries (9).

Upon admission, the patient's vital signs were stable, with a pulse of $90 \mathrm{BPM}$ and a blood pressure of $107 / 66 \mathrm{mmHg}$. The abdomen was soft with no rebound tenderness. An obstetric examination suggested that she was in good condition, with a uterine height of $28 \mathrm{~cm}$, an abdominal circumference of $90 \mathrm{~cm}$ and a FHR of 130 times per minute. Fetal movement was palpable. However, abdominal color ultrasonography suggested a uterine defect, an intrauterine single live fetus and fetal leg entrapment (Figure 1). There was a continuous interruption of the myometrium close to the left corner of the uterus. The left horn of the uterus was bulging (size: $9.8 \mathrm{~cm} \times$ $9.3 \mathrm{~cm}$ ) with no echo; fetal limbs were identifiable within the uterus. These results strongly suggested that the patient had experienced a uterine rupture.

Following hospital admission, we performed a Cesarean section to terminate the pregnancy. A male baby weighing 1,440 $\mathrm{g}$ was delivered, with Apgar scores of 8 and 9 at 1 and 5 minutes, respectively. The left leg of the neonate was blue and slightly swollen. Thus, we transferred the neonate to the Neonatology Department to receive further treatment. During the Cesarean section, we observed a small amount of bloody fluid in the abdominal cavity. We also observed an amniotic sac extrusion in the left corner of the uterus; this extrusion contained the fetal limbs (Figure 2). After delivering the fetus, we injected 20 units of oxytocin into the muscle of the uterine wall. The placenta and fetal membranes were completely delivered, and the incision in the lower uterine segment was sutured.

A large defect was identified in the left uterine horn, due to rupture of the posterior uterus (Figure 3). The old tissue was removed and the defect was repaired. Further inspection revealed an otherwise normal uterus. The patient coped well intraoperatively and recovered well postoperatively. Support and symptomatic treatments were provided to prevent infection and promote contractions. The patient was discharged from hospital on postoperative 


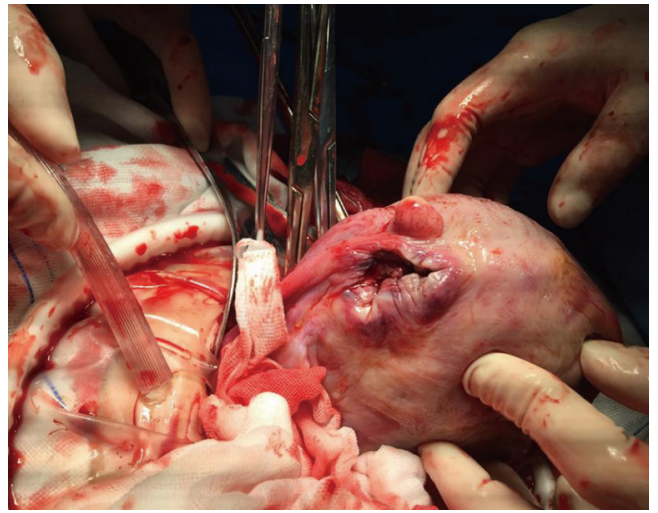

Figure 3 Photograph showing the removed placenta and the membrane. The rupture port is retracted and is approximately $6 \mathrm{~cm}$ long.

day 3 with grade II healing of the abdominal wound.

\section{Discussion}

Spontaneous rupture is a serious obstetric complication that can endanger the lives of both the mother and fetus. There are numerous causes of spontaneous rupture, including a scarred uterus, obstructive dystocia, improper use of drugs to induce uterine contractions, Cesarean section, myomectomy, uterine horn resection and uterine plasty. However, the most common factor is uterine scarring; a recent study found a statistical correlation between uterine rupture and previous Cesarean section (2). Patients with a history of Cesarean section were associated with a 9.94 times higher risk of experiencing a uterine rupture than patients who had not undergone a previous Cesarean section (2). Furthermore, obstructive dystocia, obstetric operation injury, improper use of drugs to promote uterine contractions, multiple pregnancies and uterine malformations are also linked to a high risk of spontaneous rupture. A previous study reported that the use of prostaglandin during labor is associated with a greater risk of uterine rupture (10). Another study, by Kotoulová et al., also reported that Cushing syndrome can lead to spontaneous rupture during pregnancy (11). The present case was very rare, because rupture usually occurs during the course of labor or during the third trimester before term. Reaching an accurate pre-operative diagnosis was a particular challenge in our case. The clinical features of typical uterine rupture include severe abdominal pain, vaginal bleeding, maternal low blood volume shock and massive hemorrhage (10). Once a uterus ruptures, the flow of amniotic fluid and blood are expected to cause acute abdominal tearing pain with tenderness and rebound tenderness. Hypovolemic shock has also been described in some cases. However, uterine rupture is usually not diagnosed until after the infant is delivered.

The case described here is unusual because the patient did not present with the typical clinical symptoms. She had no acute pain in her abdomen and color ultrasonography showed no obvious abnormalities. It is possible that spontaneous uterine rupture could sometimes result in only mild abdominal pain. This is because the embryolemma does not break following the uterine rupture but, instead, part of it bulges into the enterocoelia, leading to the flow of a small amount of blood into the enterocoelia. Such blood flow would be slow when the rupture occurs in the left uterine wall, in the proximity of the cornua uteri; consequently, the clinical manifestations of these injuries are not obvious.

Consequently, we present the current case to highlight the need for physicians to pay attention for spontaneous rupture. Proper action on time can help to avoid further maternal and fetal consequences. If rupture is not accurately diagnosed, valuable treatment time is lost. Thus, uterine rupture may be suspected in cases where the patient complains of durative abdominal pain, either in early or late gestation, even without intra-abdominal hemorrhage and vagina bleeding.

One of the limitations is that the patient had her 2 previous abortion surgeries performed in another hospital and it had been quite long ago, we are unable to contact the attending physician and obtain the details regarding the specific procedure done.

\section{Acknowledgments}

Funding: This work was supported by grants from the National Natural Science Foundation of China (81772772, 81302242), Capital construction fund of Jilin development and Reform Commission (2019C051-7), Jilin Medical and health talents special project (2019SCZT047).

\section{Footnote}

Conflicts of Interest: All authors have completed the ICMJE uniform disclosure form (available at http://dx.doi. org/10.21037/gs.2020.02.16). The authors have no conflicts of interest to declare. 
Ethical Statement: The authors are accountable for all aspects of the work in ensuring that questions related to the accuracy or integrity of any part of the work are appropriately investigated and resolved. Written informed consent was obtained from the patient for publication of this Case report and any accompanying images.

Open Access Statement: This is an Open Access article distributed in accordance with the Creative Commons Attribution-NonCommercial-NoDerivs 4.0 International License (CC BY-NC-ND 4.0), which permits the noncommercial replication and distribution of the article with the strict proviso that no changes or edits are made and the original work is properly cited (including links to both the formal publication through the relevant DOI and the license). See: https://creativecommons.org/licenses/by-nc-nd/4.0/.

\section{References}

1. Revicky V, Muralidhar A, Mukhopadhyay S, et al. A case series of uterine rupture: lessons to be learned for future clinical practice. J Obstet Gynaecol India 2012;62:665-73.

2. Abebe F, Mannekulih E, Megerso A, et al. Determinants of uterine rupture among cases of Adama city public and private hospitals, Oromia, Ethiopia: a case control study. Reprod Health 2018;15:161.

3. Sweeten KM, Graves WK, Athanassiou A. Spontaneous rupture of the unscarred uterus. Am J Obstet Gynecol

Cite this article as: Tan WX, Lv XJ, Yang RQ, Zhao BZ, Chen JY, Wu S, Zhao YH, Cui MH. Spontaneous rupture of an unscarred uterus with an intact amniotic sac extrusion and fetal leg entrapment at 28 gestational weeks: a case report. Gland Surg 2020;9(2):459-462. doi: 10.21037/gs.2020.02.16
1995;172:1851-5.

4. Tarney CM, Whitecar P, Sewell M, et al Rupture of an unscarred uterus in a quadruplet pregnancy. Obstet Gynecol 2013;121:483-5.

5. Surico D, Amadori R, Vigone A, et al. Successful delivery after surgical repair of uterine rupture at 15 weeks of gestation: case report and brief review. Eur J Obstet Gynecol Reprod Biol 2016;204:5-8.

6. Kieser KE, Baskett TF. A 10-year population-based study of uterine rupture. A 10-year population-based study of uterine rupture. Obstet Gynecol 2002; 100:749-53.

7. Millar WG. A case of silent antepartum rupture of the uterus. Br Med J 1961;2:218-9.

8. Lydon-Rochelle M, Holt VL, Easterling TR, et al. Risk of uterine rupture during labor among women with a prior cesarean delivery. N Engl J Med 2001;345:3-8.

9. Kotoulová M, Mikysková I, Dušková J, et al. Adrenocortical oncocytoma presenting as Cushing's syndrome in pregnancy with spontaneous postpartum uterine rupture. Ceska Gynekol 2016;81:228-32.

10. Woo JY, Tate L, Roth S, et al. Silent spontaneous uterine rupture at 36 weeks of gestation. Case Rep Obstet Gynecol 2015;2015:596826.

11. Kotoulová M, Mikysková I, Dušková J, et al. Adrenocortical oncocytoma presenting as Cushing's syndrome in pregnancy with spontaneous postpartum uterine rupture. Ceska Gynekol 2016;81:228-32. 